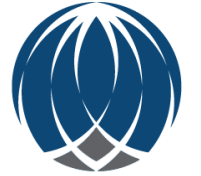

A L L E N

BRAIN ATLAS

MOUSE BRAIN

\title{
CEREBELLAR CORTEX, PURKINJE CELL LAYER (CBXpu)
}

\author{
Emily M. Wright, Lydia L. Ng and Angela L. Guillozet-Bongaarts
}

\section{Introduction}

This report contains a summary of expression patterns for genes that are enriched in the Purkinje cell layer (CBXpu) of the cerebellum. All data is derived from the Allen Brain Atlas (ABA) in situ hybridization mouse project. The structure's location and morphological characteristics in the mouse brain are described using the Nissl data found in the Allen Reference Atlas. Using an established algorithm, the expression values of the CBXpu were compared to the values of its larger parent structure, in this case the cerebellar cortex, for the purpose of extracting regionally selective gene expression data. The highest ranking genes were manually curated and verified. 50 genes were then selected and compiled for expression analysis. The experimental data for each gene may be accessed via the links provided; additional data in the sagittal plane may also be accessed using the ABA. A gene ontology table (derived from DAVID Bioinformatics Resources 2007) is also included, highlighting possible functions of the 50 genes selected for this report.

To read more about how our 50 Select Genes list is derived, please refer to the Fine Structure Annotation white paper.

Allen Reference Atlas Coronal Levels: 101-131

Allen Reference Atlas Sagittal Levels: 1-21

Shown below is a plate from the Allen Reference Atlas, depicting the Purkinje cell layer (118):

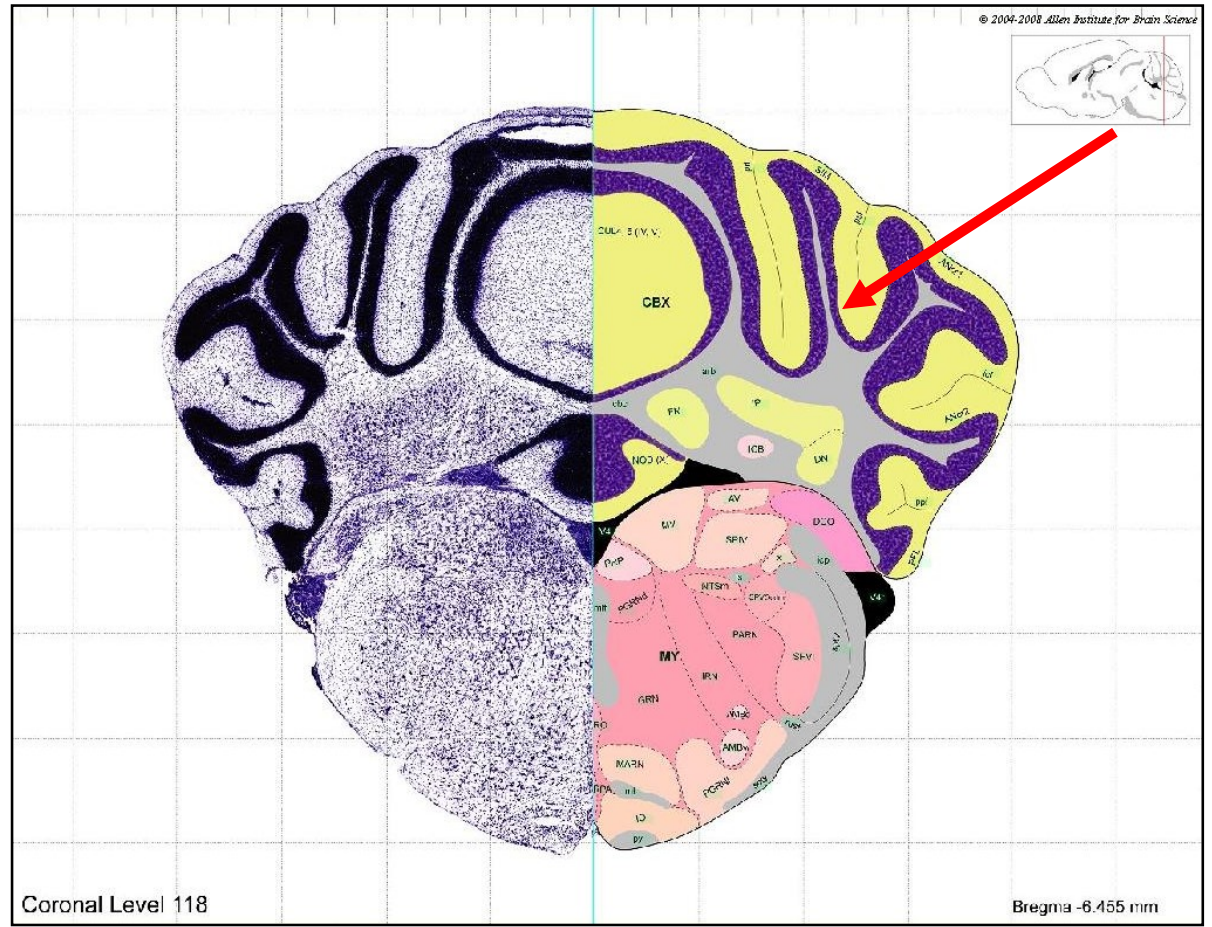




\section{Description of Structure:}

LOCATION and STRUCTURAL ANATOMY:

The hierarchical relationship within the brain is depicted below in the structure legend. The Allen Reference Atlas (based on Nisslstained sections scanned at 10X) was the primary resource for the following descriptions.

For additional information please refer to the Allen Reference Atlas white paper. BrainInfo houses a search engine that allows searches for structure name aliases.

The Purkinje cell layer is situated between the molecular and granule cell layers of the cerebellar cortex. It contains a monolayer of Purkinje cells, GABA-ergic projection neurons noted for their large cell bodies. In addition to Purkinje cells, this cell layer also contains Bergman glia, specialized astrocytes within the cerebellum that play a role in development. These specialized glia can be differentiated from Purkinje cells by their projections around the Purkinje cells, often radiating into the molecular cell layer of the cerebellar cortex.

The Purkinje cell layer is present throughout all subdivisions of the cerebellar cortex. In the Allen Reference Atlas (ARA), the Purkinje cell layer is depicted as a dotted line superimposed between the molecular and granule cell layer. However, on the Nissl-stained sections within the ARA, individual Purkinje cells are difficult to identify due to their close apposition with the darkly staining, cell-dense granular cell layer.

The appearance and location of the Purkinje cell layer can be appreciated on the following two pages. Nissl-stained sections and Allen Reference Atlas plates reveal the cytoarchitecture and extent of the Purkinje cell layer, and its location in relation to surrounding structures.

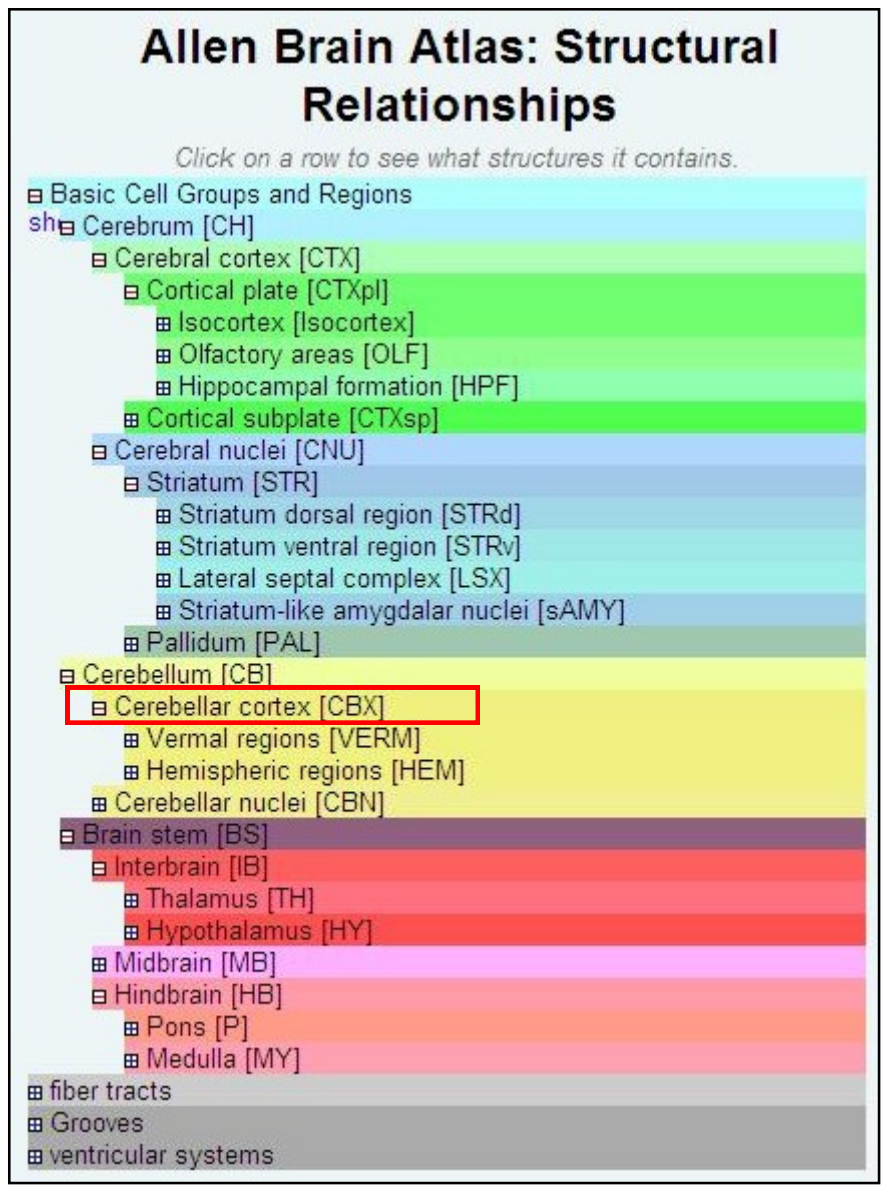




\section{Atlas and Nissl: Coronal:}

\section{Rostral}
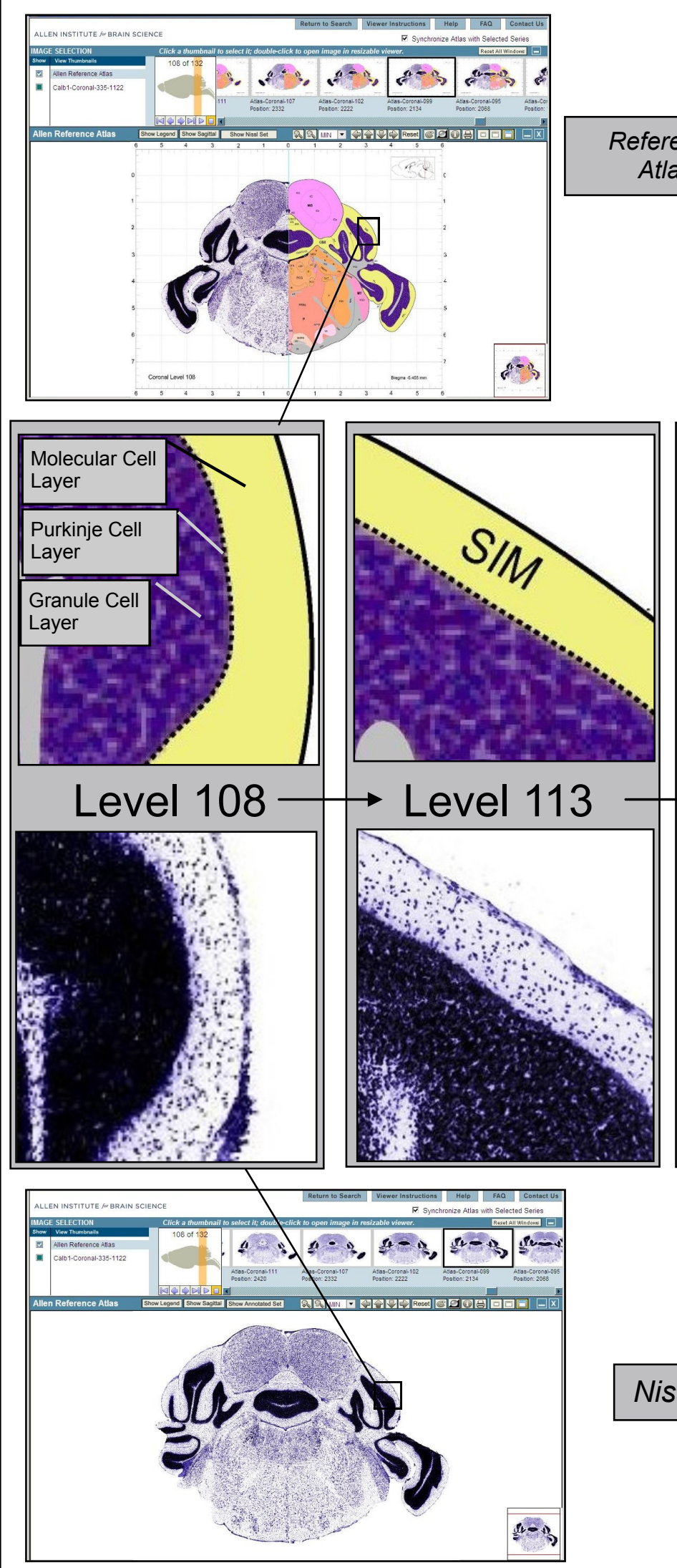

Caudal

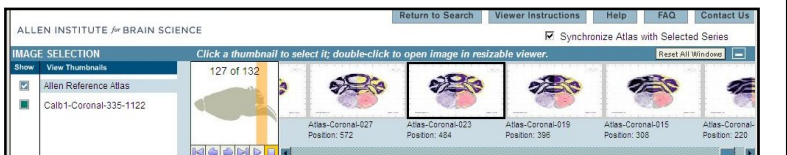
Atlas
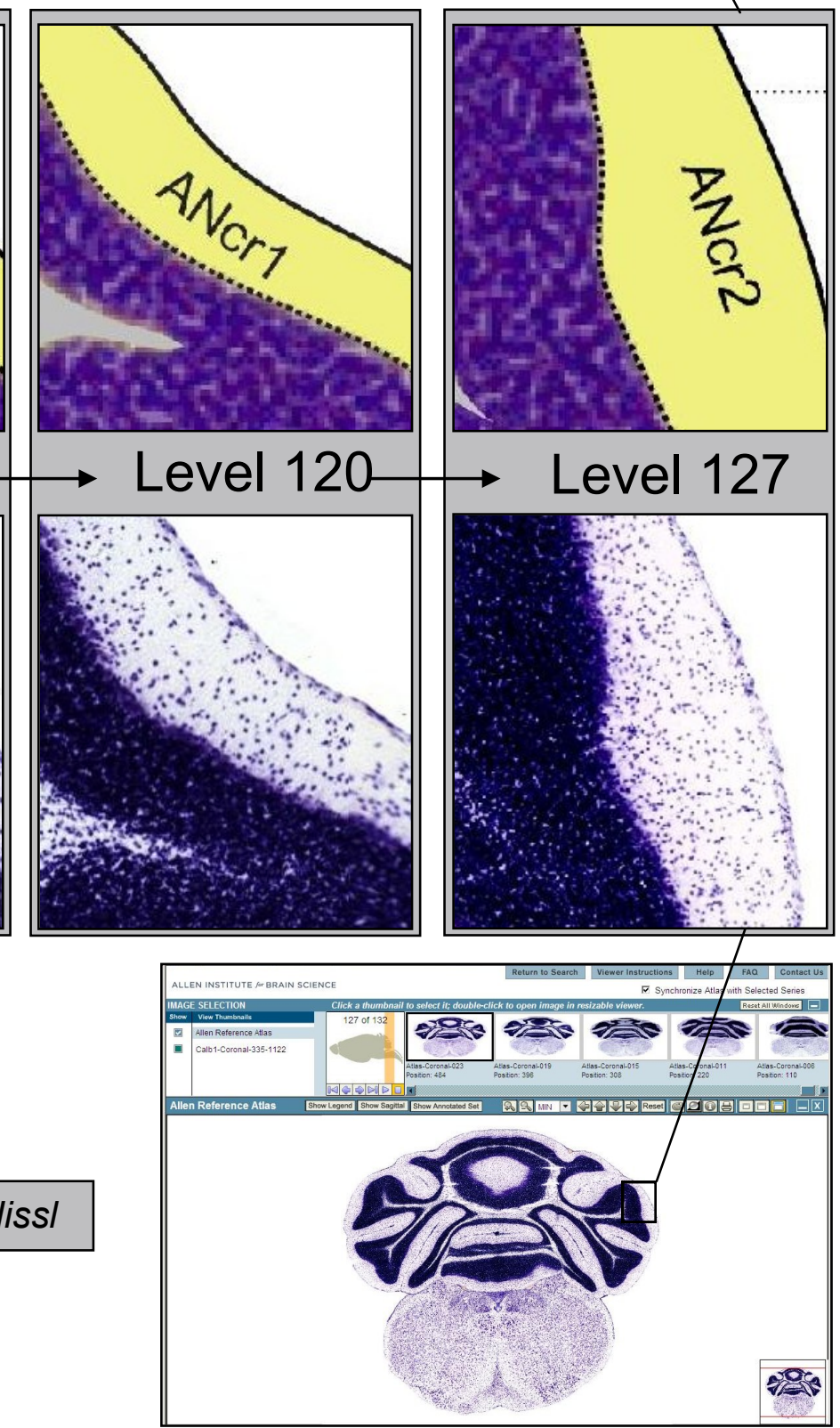


\section{Atlas and Nissl:}

Sagittal:

Lateral

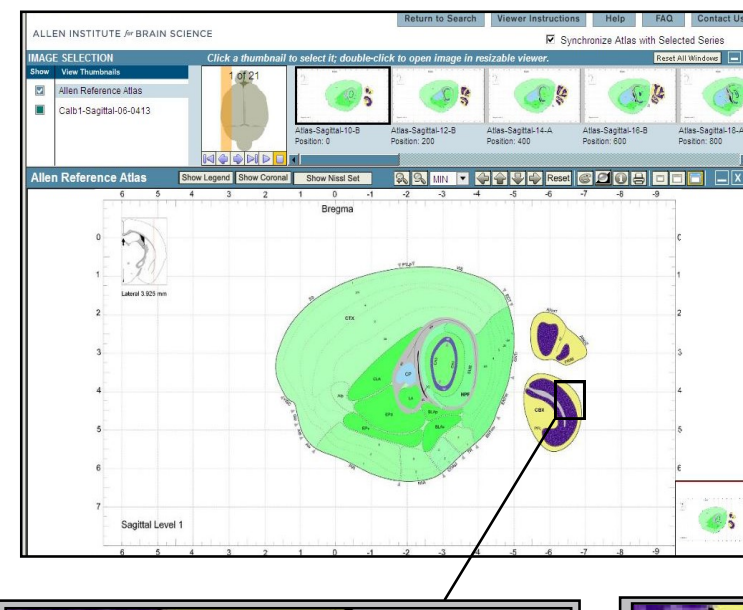

\section{Medial}

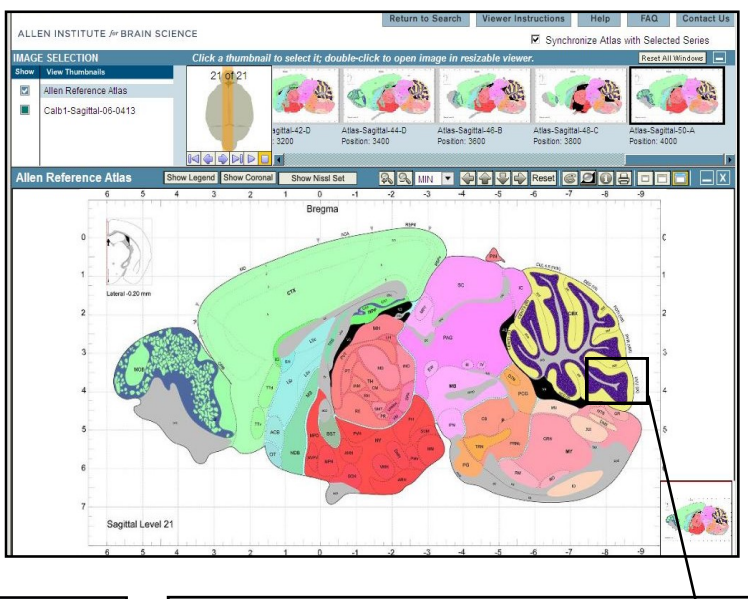

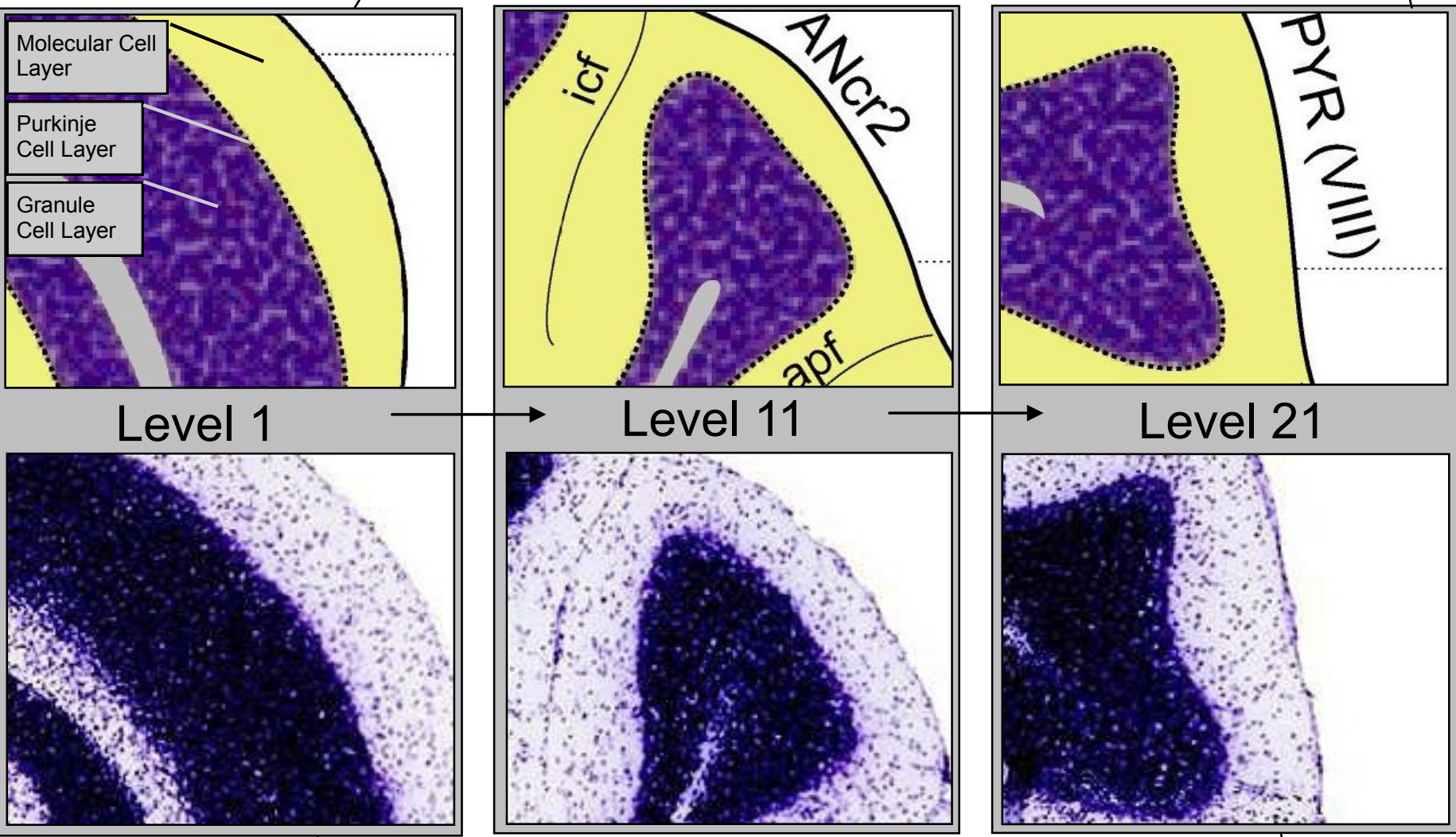
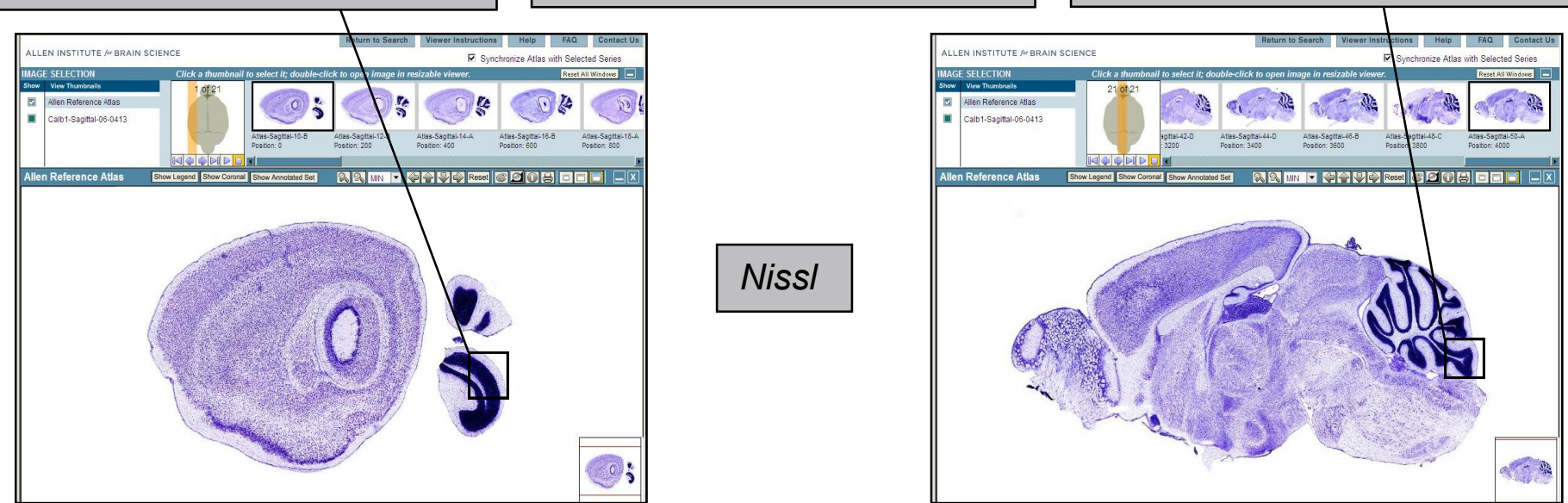
The in situ hybridization (ISH) data below presents the Purkinje cell layer's anatomical and cytoarchitectural characteristics in the con text of actual gene expression. In addition to presenting molecularly defined borders, ISH gene expression patterns also aid in phenotyping cell populations that otherwise can not be differentiated on purely morphological grounds. The 50 genes in this section were selected based on a mathematical algorithm to identify gene expression patterns that allow selective identification of the Purkinje cell layer. The gene expression patterns were then verified manually. As such, these genes do not represent the only genes found in this structure, genes specific to this structure, or genes expressing at the highest level within this structure.

Please refer to our protocol in the Data Production Processes white paper.

To read about heat map conversion, refer to the Informatics Data Processing white paper.

The expression data presented can be further explored, in coronal and sagittal planes, at brain-map.org.

The expression patterns found with these 50 Select Genes have been divided into three categories. The majority of genes examined were expressed in the Purkinje cells; some of these genes labeled only the Purkinje cells (the first category), while others labeled additional cells in the cerebellar cortex (the second category). Within the second category, these genes most often labeled golgi cells within the granule layer, although a few genes labeled interneurons within the molecular cell layer. Finally, a third group of six genes is presented that label the Bergman glia but not the Purkinje cells.

While the Purkinje cells are hard to identify on the Nissl images presented in the ABA, they are clearly demarcated with these genes. Bergman glia, which are interdigitated between Purkinje cells and difficult to distinguish from the Purkinje cells on Nissl stained sections, were easily identified in the third category, as the Purkinje cells themselves did not label, leaving a negative impression of these cells within the Purkinje cell layer.

\begin{tabular}{|l|l|l|l|}
\hline \multicolumn{2}{|l|}{ Cellular density expression key } & \multicolumn{2}{l|}{ Cellular intensity expression key } \\
\hline None & No expression & No color & Very low intensity \\
\hline Sparse & Very few cells expressing & Blue & Low intensity \\
\hline Scattered & Less than $10 \%$ of cells expressing in scattered pattern & Green & Medium intensity \\
\hline Medium & $10-80 \%$ of cells expressing & Yellow & High intensity \\
\hline High & Greater than $80 \%$ of cells expressing & Red & Very high intensity \\
\hline
\end{tabular}

To view the heat map at brain-map.org, right click on the ISH image and select "Show Expression Analysis."

ISH DATA The images below were selected to highlight various expression patterns of the Purkinje cell layer.

\section{ISH}

\section{Gng13}

\section{Coronal:}

Gng13 shows expression that is fairly restricted to Purkinje cells, but no labeling in the other layers of the cerebellar cortex.

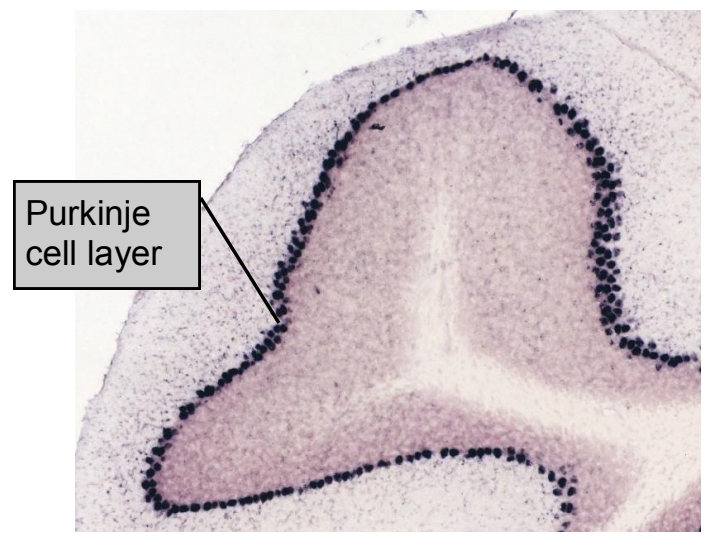

Heat Map

Gng13

\section{Coronal:}

The high density and very high intensity of Gng13 expression, restricted to the Purkinje cells, is highlighted by the heat map image.

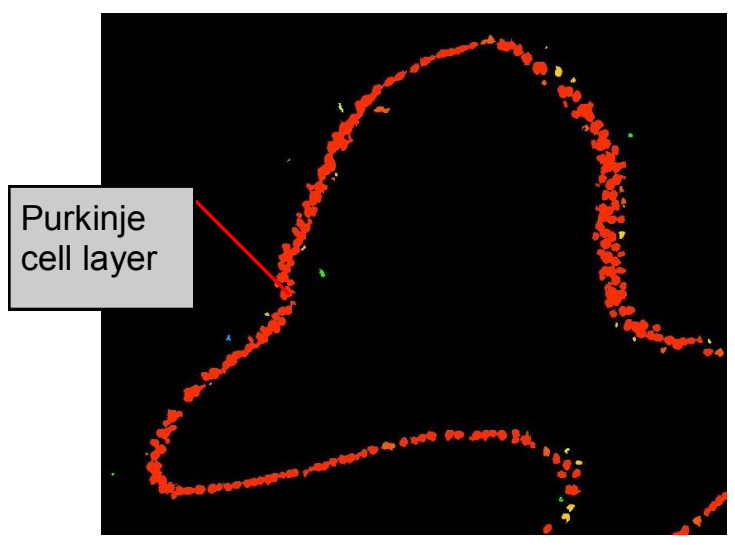


ISH

Abhd3

Coronal: $A b h d 3$ is expressed in both the Purkinje cells as well as Golgi II cells in the granule cell layer.
Heat map

Abhd3

Coronal: The heat map image reveals that $A b h d 3$ expression in the granule cell layer is less intense than the expression in the Purkinje cells.

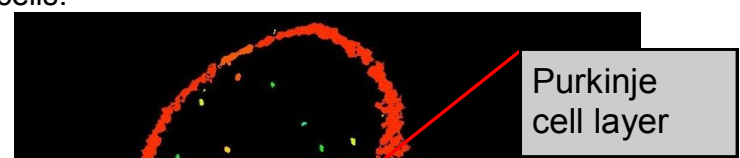

\section{Heat map}

Ppap2b

Coronal: The absence of Ppap2b expression in Purkinje cells is evident in the heat map image.

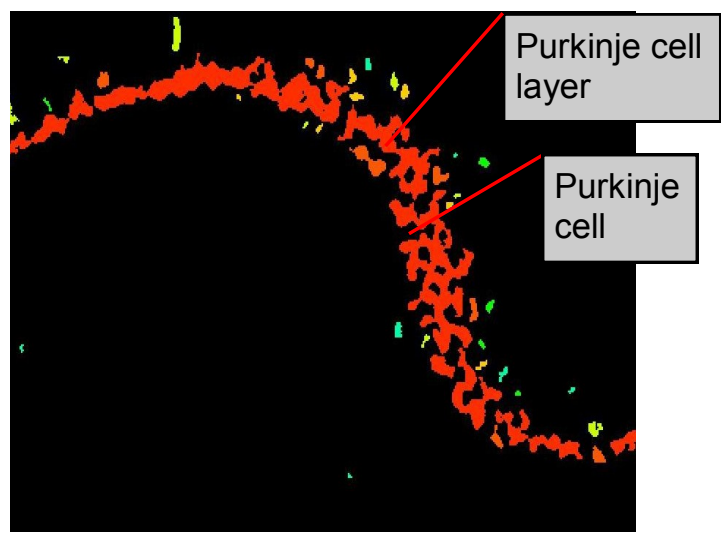




\section{SELECT GENES:}

This gene list was generated by manual curation of an algorithmically derived list that compared gene expression values of the Purkinje cell layer to those of the cerebellum. Categories of expression are subjectively grouped by relative expression characteristics.

Curation of 50 Select Genes List: June 2007

\section{Expression Restricted to Purkinje cells}

\begin{tabular}{|c|c|c|c|}
\hline Number & Gene Symbol & Gene Name & Expression Pattern \\
\hline 1 & TC1568100 & TIGR MGI TC1568100 & High density, very high intensity \\
\hline 2 & Id2 & inhibitor of DNA binding 2 & High density, very high intensity \\
\hline 3 & Cpne2 & copine II & High density, very high intensity \\
\hline 4 & Gsbs & G substrate & High density, very high intensity \\
\hline 5 & 3110001A13Rik & RIKEN cDNA $3110001 \mathrm{~A} 13$ gene & High density, very high intensity \\
\hline 6 & Pcsk6 & proprotein convertase subtilisin/kexin type 6 & High density, very high intensity \\
\hline 7 & 0610007P14Rik & RIKEN cDNA 0610007P14 gene & High density, very high intensity \\
\hline 8 & Grid2ip & $\begin{array}{l}\text { glutamate receptor, ionotropic, delta } 2 \text { (Grid2) in- } \\
\text { teracting protein } 1\end{array}$ & High density, very high intensity \\
\hline 9 & Gng13 & guanine nucleotide binding protein 13, gamma & High density, very high intensity \\
\hline 10 & Slc35b1 & solute carrier family 35, member B1 & High density, very high intensity \\
\hline 11 & |tpr1 & inositol 1,4,5-triphosphate receptor 1 & High density, very high intensity \\
\hline 12 & Baiap2 & $\begin{array}{l}\text { brain-specific angiogenesis inhibitor 1-associated } \\
\text { protein } 2\end{array}$ & High density, very high intensity \\
\hline 13 & Dgkh & diacylglycerol kinase, eta & High density, very high intensity \\
\hline 14 & Grik1 & glutamate receptor, ionotropic, kainate 1 & High density, very high intensity \\
\hline 15 & Creg1 & cellular repressor of E1A-stimulated genes 1 & High density, very high intensity \\
\hline $1 . \mathrm{TC156}$ & 8100 & \begin{tabular}{|l|l}
$\underline{2 . l d} 2$ \\
\end{tabular} & 3.Cpne2 \\
\hline 4.Gsbs & & $\underline{\text { 5.3110001A13Rik }}$ & 6.Pcsk6 \\
\hline
\end{tabular}




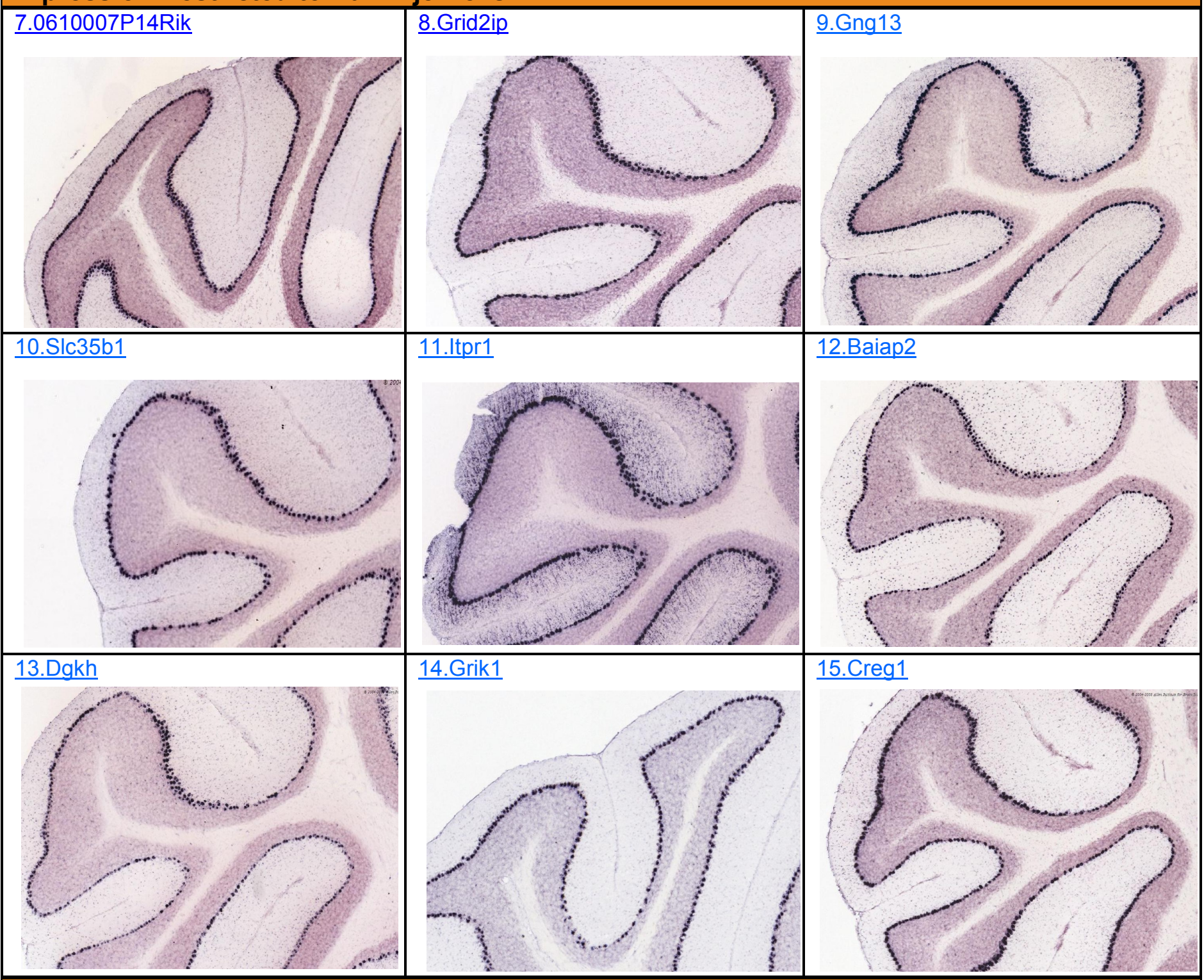

\section{Enriched Expression in Purkinje Cells and Interneurons}

\begin{tabular}{|l|l|l|l|}
\hline \hline 16 & Abhd3 & abhydrolase domain containing 3 & High density, very high intensity \\
\hline 17 & Arhagap26 & Rho GTPase activating protein 26 & High density, very high intensity \\
\hline 18 & Dpp10 & dipeptidylpeptidase 10 & High density, very high intensity \\
\hline 19 & Ryr1 & ryanodine receptor 1, skeletal muscle & High density, very high intensity \\
\hline 20 & Steap2 & six transmembrane epithelial antigen of prostate 2 & High density, very high intensity \\
\hline 21 & Gabbr2 & gamma-aminobutyric acid (GABA) B receptor 2 & High density, very high intensity \\
\hline 22 & Cerk & ceramide kinase & High density, very high intensity \\
\hline 23 & Lrrn2 & leucine rich repeat protein 2, neuronal & High density, very high intensity \\
\hline 24 & Nefh & neurofilament, heavy polypeptide & High density, very high intensity \\
\hline 25 & Atp2a2 & ATPase, Ca++ transporting, cardiac muscle, slow & \\
\hline 26 & Im6sf1 & tritch 2 & High density, very high intensity \\
\hline 27 & Nell2 & NEL-like 2 (chicken) & High density, very high intensity \\
\hline 28 & Hapln4 & hyaluronan and proteoglycan link protein 4 & High density, very high intensity \\
\hline 29 & Hpca & hippocalcin & High density, very high intensity \\
\hline 30 & Setd7 & SET domain containing (lysine methyltransferase) 7 & High density, very high intensity \\
\hline & & low density lipoprotein receptor-related protein 8, & High density, very high intensity \\
\hline 31 & Lrp8 & apolipoprotein E receptor \\
\hline 32 & Grid2 & glutamate receptor, ionotropic, delta 2 & \\
\hline & & High density, very high intensity \\
\hline
\end{tabular}




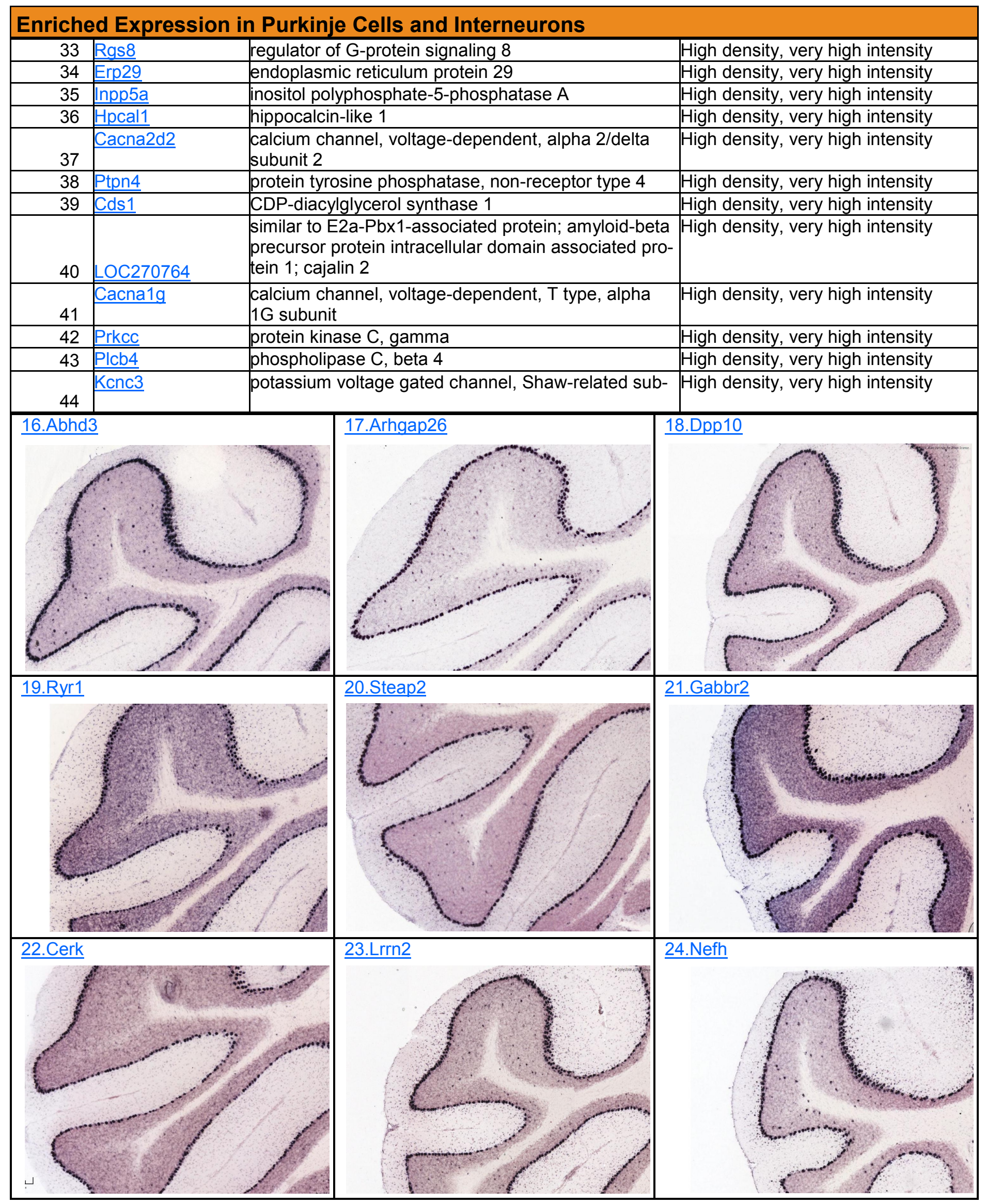




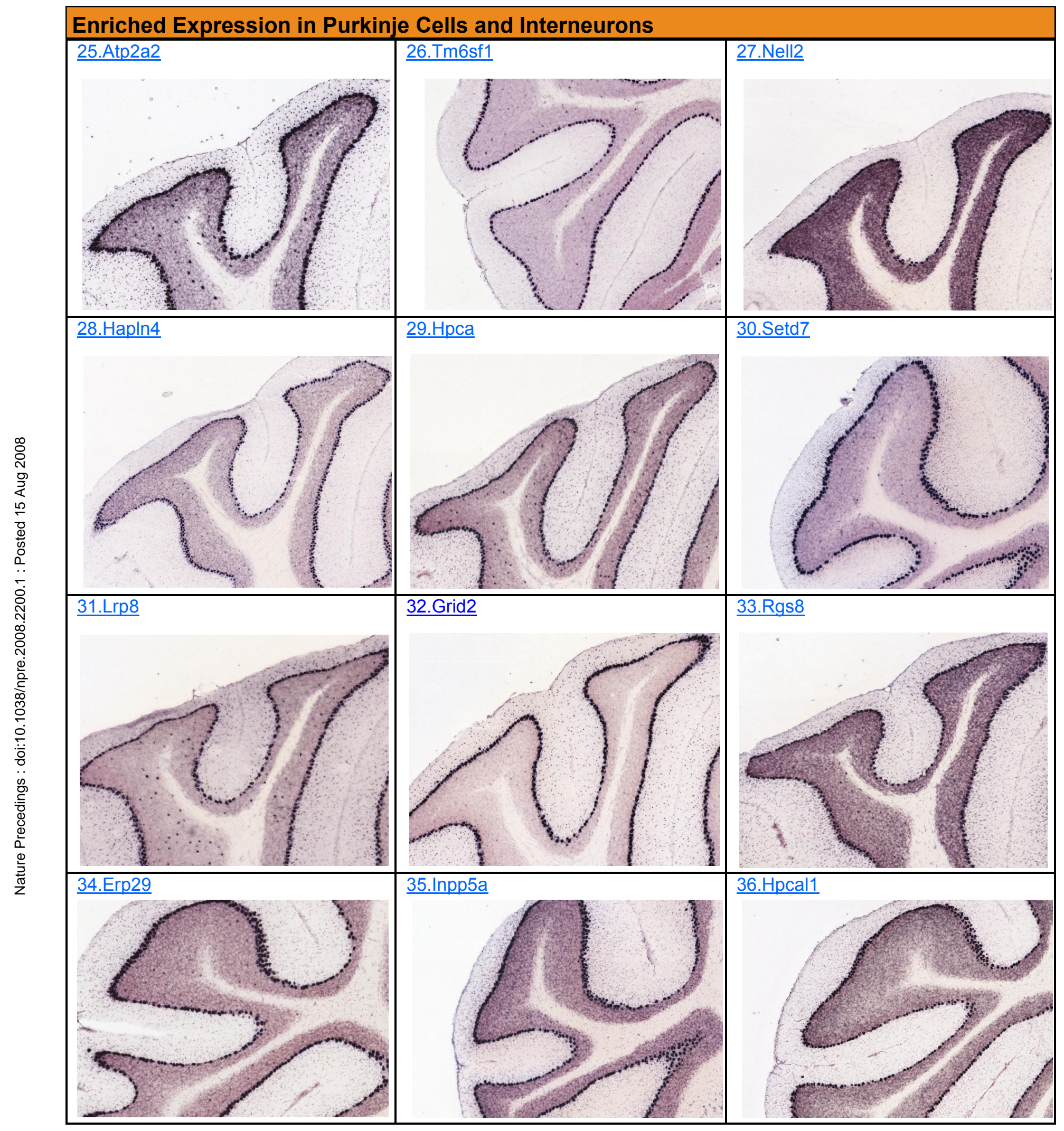




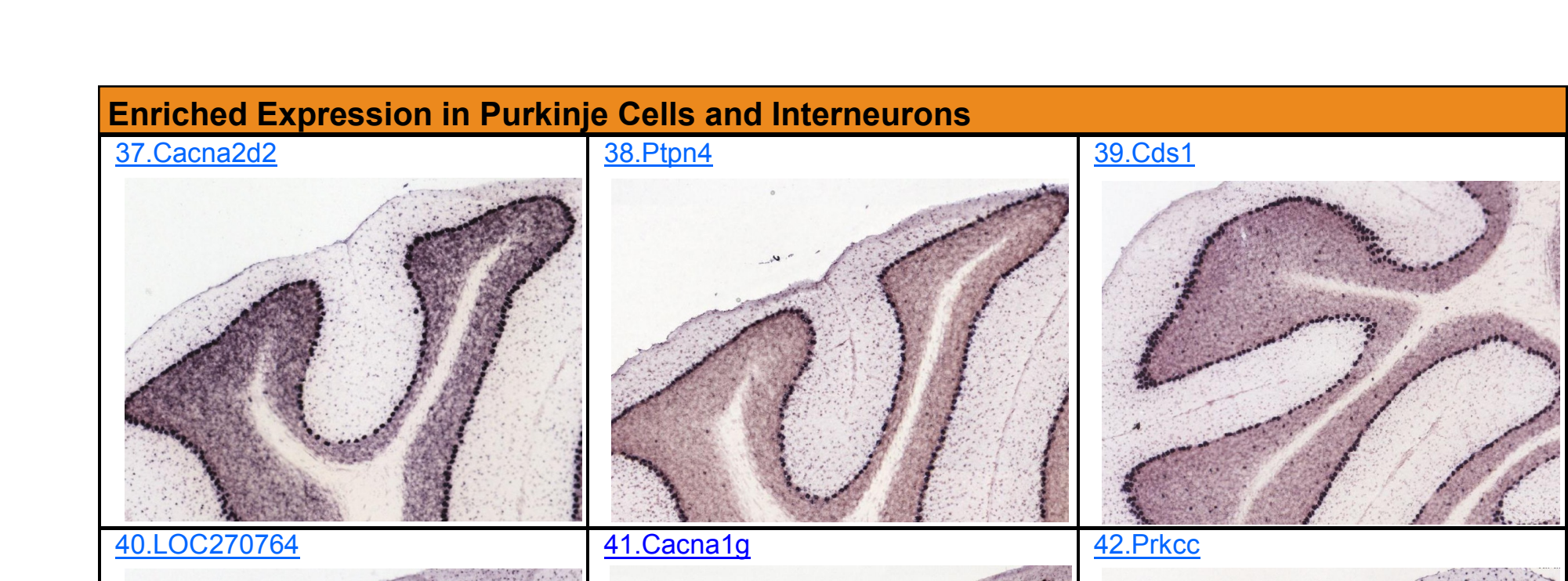

NI vI WU

nos

SA GA 


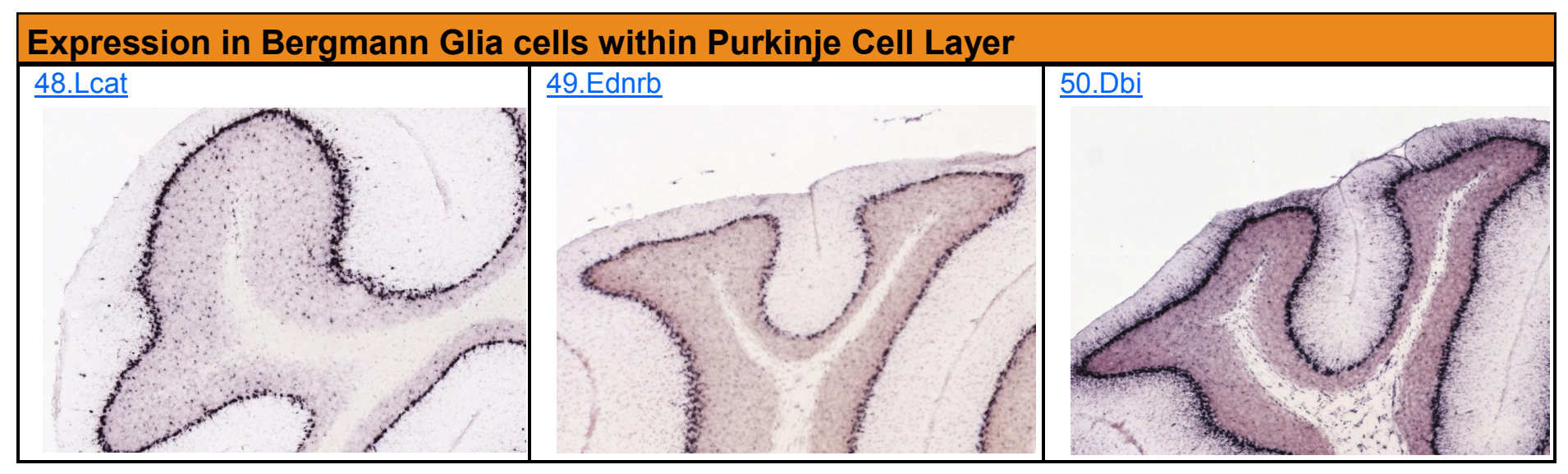




\section{Gene Ontology (GO) Analysis:}

\section{GO TABLE:}

Below is an ontological analysis of the 50 Select Genes, using DAVID Bioinformatics Resources. The functional terms that follow were returned using these constraints:

\begin{tabular}{|l|l|l|}
\hline Category & Definition & Constraints \\
\hline P-value & $\begin{array}{l}\text { Probability that the term is over-represented in this } 50 \\
\text { Select Genes list relative to the mouse genome }\end{array}$ & when p $\leq 0.05$ \\
\hline Gene Count & $\begin{array}{l}\text { The minimum number of genes that must fall into an onto- } \\
\text { logical category to be considered a group }\end{array}$ & 5 genes per term group \\
\hline GO Level & $\begin{array}{l}\text { The level of functional specificity for GO functional cate- } \\
\text { gories: Molecular Function (mf), Biological Process (bp) } \\
\text { and Cellular Components (cc) }\end{array}$ & Level GO_All \\
\hline \# of DAVID IDs & Number of unique DAVID gene IDs from user's input list & 48 DAVID gene IDs/ 50 input genes \\
\hline
\end{tabular}

Date of table completion: June 2008

\begin{tabular}{|c|c|c|c|c|}
\hline GO Category & GO Term & Gene Count & $\%$ of Genes & $p$-value \\
\hline GOTERM_MF_ALL & calcium ion binding & 12 & $25.00 \%$ & $4.33 \mathrm{E}-06$ \\
\hline GOTERM_BP_ALL & di-, tri-valent inorganic cation transport & 6 & $12.50 \%$ & $3.24 \mathrm{E}-05$ \\
\hline GOTERM_MF_ALL & gated channel activity & 7 & $14.58 \%$ & $4.62 \mathrm{E}-05$ \\
\hline GOTERM_MF_ALL & metal ion transmembrane transporter activity & 7 & $14.58 \%$ & $7.45 \mathrm{E}-05$ \\
\hline GOTERM_CC_ALL & membrane fraction & 8 & $16.67 \%$ & $1.78 \mathrm{E}-04$ \\
\hline GOTERM_BP_ALL & calcium ion transport & 5 & $10.42 \%$ & $1.89 \mathrm{E}-04$ \\
\hline GOTERM_MF_ALL & ion channel activity & 7 & $14.58 \%$ & $1.97 \mathrm{E}-04$ \\
\hline GOTERM_MF_ALL & substrate specific channel activity & 7 & $14.58 \%$ & $2.32 \mathrm{E}-04$ \\
\hline GOTERM_MF_ALL & cation channel activity & 6 & $12.50 \%$ & $3.59 \mathrm{E}-04$ \\
\hline GOTERM_MF_ALL & passive transmembrane transporter activity & 7 & $14.58 \%$ & $3.75 \mathrm{E}-04$ \\
\hline GOTERM_MF_ALL & channel activity & 7 & $14.58 \%$ & $3.75 \mathrm{E}-04$ \\
\hline GOTERM_CC_ALL & cell fraction & 8 & $16.67 \%$ & $3.89 \mathrm{E}-04$ \\
\hline GOTERM_BP_ALL & ion transport & 9 & $18.75 \%$ & $4.45 \mathrm{E}-04$ \\
\hline GOTERM_MF_ALL & substrate-specific transmembrane transporter activity & 9 & $18.75 \%$ & $5.29 \mathrm{E}-04$ \\
\hline GOTERM_BP_ALL & metal ion transport & 7 & $14.58 \%$ & $5.59 \mathrm{E}-04$ \\
\hline GOTERM_MF_ALL & ion transmembrane transporter activity & 8 & $16.67 \%$ & 0.001158 \\
\hline GOTERM_MF_ALL & transmembrane transporter activity & 9 & $18.75 \%$ & 0.001375 \\
\hline GOTERM_MF_ALL & cation transmembrane transporter activity & 7 & $14.58 \%$ & 0.001413 \\
\hline GOTERM_BP_ALL & cation transport & 7 & $14.58 \%$ & 0.001656 \\
\hline GOTERM_CC_ALL & endoplasmic reticulum & 8 & $16.67 \%$ & 0.002352 \\
\hline GOTERM_MF_ALL & substrate-specific transporter activity & 9 & $18.75 \%$ & 0.002402 \\
\hline GOTERM_MF_ALL & transporter activity & 10 & $20.83 \%$ & 0.004188 \\
\hline GOTERM_BP_ALL & lipid metabolic process & 7 & $14.58 \%$ & 0.004372 \\
\hline GOTERM_BP_ALL & establishment of localization & 13 & $27.08 \%$ & 0.019275 \\
\hline GOTERM_BP_ALL & intracellular signaling cascade & 8 & $16.67 \%$ & 0.023088 \\
\hline GOTERM_MF_ALL & metal ion binding & 16 & $33.33 \%$ & 0.024839 \\
\hline GOTERM_MF_ALL & cation binding & 15 & $31.25 \%$ & 0.029425 \\
\hline GOTERM_MF_ALL & ion binding & 16 & $33.33 \%$ & 0.029561 \\
\hline GOTERM_BP_ALL & cell communication & 17 & $35.42 \%$ & 0.029929 \\
\hline GOTERM_CC_ALL & plasma membrane & 11 & $22.92 \%$ & 0.035826 \\
\hline GOTERM_BP_ALL & transport & 12 & $25.00 \%$ & 0.038328 \\
\hline GOTERM_MF_ALL & transferase activity, transferring phosphorus-containing groups & 7 & $14.58 \%$ & 0.042668 \\
\hline GOTERM_BP_ALL & Iocalization & 13 & $27.08 \%$ & 0.044765 \\
\hline GOTERM_CC_ALL & cytoplasm & 21 & $43.75 \%$ & 0.045585 \\
\hline GOTERM_BP_ALL & cellular lipid metabolic process & 5 & $10.42 \%$ & 0.046498 \\
\hline
\end{tabular}

Glynn Dennis Jr., Brad T. Sherman, Douglas A. Hosack, Jun Yang, Michael W. Baseler, H. Clifford Lane, Richard A. Lempicki. "DAVID: Database for Annotation, Visualization, and Integrated Discovery." Genome Biology. 2003 4(5): P3. 


\section{Anatomy}

- The Purkinje layer, situated between the molecular and granule cell layers of the cerebellar cortex, contains two types of cells: Purkinje cells, a monolayer of large neurons, and Bergman glia, a specialized type of astrocyte.

- The Purkinje cell layer is present in all subdivisions of the cerebellum, including the vermis, the hemispheres, and the flocculonodular lobe.

- This layer is evident in both coronal and sagittal views, but is hard to discern on the images of Nissl-stained

\section{Expression Patterns of the $\mathbf{5 0}$ Select Genes} sections due to the high density and intensity of label in the granule cell layer.

- All 50 select genes have a widespread, high density and very high intensity expression pattern.

- A subset of genes examined showed expression restricted to the Purkinje cells.

- Another subset of genes showed expression that was enriched in the Purkinje cells and in multiple other cell types of the cerebellar cortex, primarily other GABA-ergic interneurons in the granule cell and the molecular cell layers.

- A final subset of the genes examined highlighted Bergman glia. In these cases, the ISH reaction product highlights the Purkinje cell layer, but the Purkinje cells themselves are devoid of label.

We encourage you to reply with any comments or questions by email to! Annotation@alleninstitute.org. To further explore the gene expression data and analytical tools referred to in this report, please access our genome-wide data set at brain-map.org.

\section{Other Tools:}

\section{NEUROBLAST:}

Many of the 50 genes listed in this report can be used to explore the NeuroBlast tool. This unique mining tool works seamlessly from within brain-map.org to produce a list of genes that share similar expression patterns to any gene in the coronal data set. Search for and select any gene, then select one of several brain regions from the NeuroBlast drop-tab to explore a ranked list of similarly expressed genes for that region.

To learn more about this function, please refer to the NeuroBlast white paper.

\section{BRAIN EXPLORER:}

To compare gene expression levels across anatomical structures in 3-D detail, download the Brain Explorer desktop application. This program is used to view gene expression in 3-D view (coronal, sagittal, horizontal and everywhere in between) across all brain structures and allows for simultaneous viewing of multiple expression profiles.

The NeuroBlast spatial homology function and an anatomic search tool are also available from within Brain Explorer to allow the user to search for and visualize genes with similar expression patterns. 